\title{
Functional mesoporous materials
}

\author{
Dongyuan Zhao $(\bowtie)$
}

Laboratory of Advanced Materials, Faculty of Chemistry and Materials, Shanghai Key Lab of Molecular Catalysis and Innovative Materials, and State Key Laboratory of Molecular Engineering of Polymers, Fudan University, Shanghai 200433, China

(C) Tsinghua University Press and Springer-Verlag GmbH Germany, part of Springer Nature 2021

The Nano Research Award, established by Nano Research together with Tsinghua University Press (TUP) and Springer Nature in 2013, is awarded for outstanding contributions in nanoscience and nanotechnology by individual scientists in all the world. The winners are selected by the Award Committee after receiving nominations. To date, the Nano Research Award has been awarded to 12 scientists. I am very honored to receive the Nano Research Award in 2020, with Prof. Rogers. The recognition is greatly an acknowledgement of the scientific advances achieved by my graduate students and postdoctoral fellows-laborious, talented, dedicated, creative, collaborative people, of the very highest caliber. The topics of this special issue span a wide range, as a powerful set of projects at the forefront of nanoscience and nanotechnology, briefly summarized by topic area as follows: nanoelectronic-photonic devices and functional mesoporous materials. Prof. Rogers has introduced the recent progresses about the nanoelectronic-photonic devices.

Besides those, a series of papers highlight the synthesis of novel mesoporous materials. Prof. An-Hui Lu from Dalian University of Technology reviews the development of the hollow structured porous carbon materials constructed by confined nanospace pyrolysis strategy in the past decade [1]. Prof. Zhen-An Qiao from Jilin University reports a versatile ligand-assisted cooperative template method to synthesize multi-shelled mesoporous hollow metal hydroxide and metal oxide nanospheres, which exhibit excellent catalytic activity for solvent-free aerobic oxidation of ethylbenzene [2]. Prof. Chengzhong Yu from East China Normal University highlights the recent progress in mesoporous resin nanobowls with optimized benzenoid-quinoid donor-acceptor conjugation and its photocatalysis performance for hydrogen peroxide $\left(\mathrm{H}_{2} \mathrm{O}_{2}\right)$ production in a sacrificial agentfree system [3]. Prof. Ben Liu from Nanjing Normal University describes a simple soft-template method to precisely engineer porous architectures of quaternary PdCuBP mesoporous nanospheres [4]. These uniform highly ordered mesoporous nanospheres exhibit excellent electrocatalytic performance toward ethanol oxidation reaction. Dr. Sheng Dai and his students from The University of Tennessee presents a research article on perfluorinated porous organic networks and their application in chlorofluorocarbons adsorption [5]. Prof. Fuxing Sun from Jilin University demonstrates the construction of two flexible cationic metal-organic frameworks with remarkable stability for $\mathrm{CO}_{2} / \mathrm{CH}_{4}$ separation [6]. Impacts of nano-scale pore structure and organic amine assembly in porous silica on the kinetics of $\mathrm{CO}_{2}$ adsorptive separation has been well studied by Prof. Xinwen Guo, Prof. Chunshan Song and coworkers from Dalian University of Technology [7].

Another research hotspot is in porous nanomaterials for catalysis, drug delivery and energy storage. Prof. Liqiang Mai from Wuhan University of Technology introduces and in-depth analyzes the various modified heterostructure electrodes with optimized electrochemical performance and wide applications [8]. These heterostructued electrodes can be nationally designed by grafting the advantages of functional materials onto the active materials. Prof. Yang Hou from Zhejiang University summarizes the latest research advances in recent developments of non-precious metal heterogeneous electrocatalysts for electroreduction of $\mathrm{CO}_{2}$ into high-value multicarbon products [9]. Prof. Nanfeng Zheng from Xiamen University proposes an unprecedented strategy to access to ultra-stable copper-hydride nanoclusters (NCs) by using bidentate N-heterocyclic carbenes as stabilizing ligands in addition to thiolates [10]. Prof. Jie Fan from Zhejiang University reports that an artificial catalyst, composing of trypsin and a calcium ion exchanged zeolite $\mathrm{Y}$ (trypsin/CaY), is capable of conducting surface-confined thrombin generation [11]. Prof. Yunfeng Lu from University of California at Los Angeles successfully develops a nanocapsule platform enabling effective delivery of miRNA systemically to the disease sites in the brain [12]. Prof. Liming Dai from the University of New South Wales reports a novel confinementsynthesis approach to layered double hydroxide nanodots anchored on carbon nanoparticles, which exhibit super-high catalytic performance for oxygen evolution reaction [13].

Over the past decades, Prof. Zhao's group has focused our researches on both the synthesis of functional mesoporous materials with controllable compositions, functionalities, and nanostructures, as well as the application of the functional mesoporous materials in various fields such as catalysis, water treatment, electrochemistry, bio-medicine, and so on. The composition and nanostructures of mesoporous materials have significant effects on their functionalities, therefore the tuning of which satisfies the demands in different application fields. However, the urgent need to achieve the controllable tuning of functional mesoporous materials' composition and nanostructures is hindered due to the limited synthetic approaches. Our group pursues the development of novel synthesis strategies and has achieved plentiful results. For the applications of functional mesoporous materials, the large pore size, high surface area, ordered mesopore channels and the highly-thermal stability render them great application potentials. For example, the large and ordered mesopore channels are beneficial to the transport of target molecules and ions. The high surface area can improve the adsorption and loading amount for target molecules and ions. These properties benefit functional mesoporous materials' applications in catalysis and energy storage fields. Yet, albeit unique mesoporous structures, 
the mesoporous materials are usually needed to be specifically fictionalized due to different application requirements. We have focused on the construction of functional mesoporous materials for many years, obtaining a series of unique functional mesoporous materials which showed excellent performance in various fields.

Up to now, we have reported a series of synthesis methods for functional mesoporous materials. For example, a generalized non-aqueous "acid-base pair" synthetic approach has been demonstrated by Prof. Zhao and co-workers, in which the formed synergistic inorganic-inorganic acid-base precursor pairs can promote the co-assembly process to obtain ordered mesostructures. Following this strategy, a group of mesoporous materials with various mesostructures and compositions have been prepared, including metal oxides and metal phosphates, which expanded and advanced the sol-gel chemistry. Prof. Zhao and co-workers also demonstrated an organic-organic assembly approach to synthesize ordered mesoporous polymers and a direct transformation to homologous carbon frameworks. Through this approach, a family of ordered mesoporous organic polymers and carbons with variable mesostructures (two-demonsional (2D) hexagonal, three-dimensional (3D) cubic structures with space groups of $\operatorname{Im} 3 m, I a 3 d, F m 3 m$ ) have been achieved. Afterward, we have also extended this strategy to synthesize mesoporous carbon spheres and vesicles with uniform diameters. The mass production of mesoporous carbons accelerates their applications in catalysis, electrochemical supercapacitors, and water-treatment fields. Recently, we have developed an "insert" super-assembly strategy to incorporate ultrasmall graphitic pencil nanodots into various ordered mesoporous frameworks. This facile and robust approach reduces the effects of the typical hydrolysis and condensation steps on the inorganic species required during the synthesis and instead co-assembles a variety of species of very diverse sizes.

The architecture of core@shell structured mesoporous nanomaterials is also of great importance. In 2008, we demonstrated a surfactant-assisted coating route to synthesize the functional core-shell ordered mesoporous silica nanoparticles with center-radially oriented mesopore channels. The method is facile and easily repeatable, many research groups in the world have used this method to construct functional core-shell nanospheres for bio-applications. We also demonstrated a kinetics-controlled coating method for the synthesis of uniform core@ $\mathrm{TiO}_{2}$ shell structures. The preferential heterogeneous nucleation and growth of mesoporous $\mathrm{TiO}_{2}$ on the surface of various cores (e.g., $\alpha-\mathrm{Fe}_{2} \mathrm{O}_{3}$ ellipsoids, $\mathrm{Fe}_{3} \mathrm{O}_{4}$ spheres, $\mathrm{SiO}_{2}$ spheres, and graphene oxide nanosheets) can be realized by precisely controlling the reaction kinetics. Later on, we discovered an "oriented assembly" strategy for the construction of a type of 2D ordered mesoporous carbon nanosheets, which offer a unique combination of flat graphene layers with large surface area, and abundant ordered mesopores as efficient ion transport pathways. This work paves a way to construct mesoporous materials based on the oriented assembly of micelles. In 2015, we extended this micelle-directed oriented assembly strategy to the construction of a series of functional mesoporous $\mathrm{TiO}_{2}$ microspheres and mesocrystals. Recently, we have combined the monomicelles-directed oriented assembly strategy and interface engineering to the synthesis of a series of core-shell, 2D, and 2D heterostructures-like mesoporous titania-based nanocomposites. Besides the symmetric mesoporous nanocomposites, series of mesoporous nanocomposites with anisotropic nanostructures on geometry, chemical composition, surface property, and functionality, etc. have been also developed in recent years, including Janus, single-hole hollow, diblock Janus, triblock Janus, engine-trailer-structured nanotrucks, multipods structures, etc. Most importantly, the obtained asymmetric nanocomposites possess unique multiple independent surfaces, compositions, functions, etc., which are ideal for selectively multiplecomponent conjugations, loading, and smart delivery system designing, etc.

Though significant advances have been made in the synthesis and applications of functional mesoporous materials, there remain several challenges to be solved. Firstly, it is still urgent to achieve precise control of the mesoporous materials' compositions, functionalities, and nanostructures. However, a versatile and simple synthetic theory is still lacking to achieve this goal due to the complex co-assembly processes of surfactants and precursors. To achieve this purpose, it is essential to better understand the underlying mechanisms in the co-assembly process from atomic to nano and micro levels. Monomicellesbased assembly method shows great promising in the precise synthesis field, in which the pore building blocks are composite micelles, not traditional surfactant/block copolymer molecules and precursors. As a result, functional mesoporous materials with well-defined symmetry and geometry can be obtained through the direct co-assembly of monomicelles. The key issue of this method is the creation of stable composite monomicelles for modular assembly. However, the development of this method is still in its primitive stage, much more work is still needed to be explored in near future.

Furthermore, the reported functional mesoporous materials are all in solid-state, it is highly desired yet challenging to construct mesoporous liquids or liquid mesoporous materials with flexible architectures. This new kind of mesoporous materials own the porosity of solid sorbents and fluid properties of liquids simultaneously. Many new interesting properties will emerge and can be expected. For example, it can unify homogeneous and heterogeneous processes that show huge application potential in separation, catalytic, energy, and biological fields. Thirdly, there is still plenty of room to further explore the applications of functional mesoporous materials deeply and widely. For example, understanding the structureperformance relationships is highly desired, which is the key for the design and practical use of functional mesoporous materials. Besides, it is crucial to explore the unique application field of functional mesoporous materials, which can well match their structural advantages. For example, the structural capabilities at the scale of a few nanometers can meet the demands of the applications emerging in large molecules involved in processes such as drug delivery and petroleum cracking, which are both critical to human society. Finally, it is important to develop new technologies for the mass production of functional mesoporous materials with diverse compositions and structures. However, except for mesoporous silica and carbon, the mass-production technology for other functional mesoporous is still lacking. The development of mass production technology will result in their more widespread application and technical advances.

\section{Reference}

[1] Zhang, R.-P.; Li, W.-C.; Hao, G.-P.; Lu, A.-H. Confined nanospace pyrolysis: A versatile strategy to create hollow structured porous carbons. Nano Res. 2021, 14, 3159-3173.

[2] Liu, J. W.; Ma, Y. L.; Zhang, L. L.; Zheng, Y. N.; Zhang, R.; Zhang, L.; Wei. F.; Qiao, Z.-A. A versatile ligand-assisted cooperative template method to synthesize multi-shelled mesoporous hollow metal hydroxide and oxide nanospheres as catalytic reactors. Nano Res. 2021, 14, 3260-3266. 
[3] Yuan, L.; Zhang, C. Q.; Wang, J.; Liu, C.; Yu, C. Z. Mesoporous resin nanobowls with optimized donor-acceptor conjugation for highly efficient photocatalytic hydrogen peroxide production. Nano Res. 2021, 14, 3267-3273.

[4] Lv, H.; Wang, Y. R.; Xu, D. D.; Liu, B. Engineering porous architectures in multicomponent $\mathrm{PdCuBP}$ mesoporous nanospheres for electrocatalytic ethanol oxidation. Nano Res. 2021, 14, 32743281.

[5] Luo, Y. L.; Yang, Z. Z.; Suo, X.; Chen, H.; Wang, T.; Wang, Z. Q.; Liu, Y. F.; Lyu, Y.; Popovs, I.; Dai, S. Robust perfluorinated porous organic networks: Succinct synthetic strategy and application in chlorofluorocarbons adsorption. Nano Res. 2021, 14, 3282-3287.

[6] Li, S. L.; Zeng, S. L.; Tian, Y. Y.; Jing, X. F.; Sun, F. X.; Zhu, G. S. Two flexible cationic metal-organic frameworks with remarkable stability for $\mathrm{CO}_{2} / \mathrm{CH}_{4}$ separation. Nano Res. 2021, 14, 3288-3293.

[7] Lou, F. J.; Zhang, G. H.; Ren, L. M.; Guo, X. W.; Song, C. S. Impacts of nano-scale pore structure and organic amine assembly in porous silica on the kinetics of $\mathrm{CO} 2$ adsorptive separation. Nano Res. 2021, 14, 3294-3302.

[8] Wu, B. K.; Luo, W.; Li, M.; Zeng, L.; Mai, L. Q. Achieving better aqueous rechargeable zinc ion batteries with heterostructure electrodes.
Nano Res. 2021, 14, 3174-3187.

[9] Chen, J. Y.; Wang, T. T.; Li, Z. J.; Yang, B.; Zhang, Q. H.; Lei, L. C.; Feng, P. Y.; Hou, Y. Recent progress and perspective of electrochemical $\mathrm{CO}_{2}$ reduction towards $\mathrm{C}_{2}-\mathrm{C}_{5}$ products over non-precious metal heterogeneous electrocatalysts. Nano Res. 2021, 14, 3188-3207.

[10] Shen, H.; Wang, L. Z.; López-Estrada, O.; Hu, C. Y.; Wu, Q. Y.; Cao, D. X.; Malola, S.; Teo, B. K.; Häkkinen, H.; Zheng, N. F. Copperhydride nanoclusters with enhanced stability by N-heterocyclic carbenes. Nano Res. 2021, 14, 3303-3306.

[11] Yu, L. S.; Yu. B.; Chen, H.; Shang, X. Q.; He, M.; Lin, M. C.; Li, D.; Zhang, W. Z.; Kang, Z. Z.; Li, J. C. et al. Highly efficient artificial blood coagulation shortcut confined on Ca-zeolite surface. Nano Res. 2021, 14, 3309-3318.

[12] Liu, C. Y.; Wen, J.; Li, D.; Qi, H. Z.; Nih, L.; Zhu, J. L.; Xu, D.; Ren, Y.; Zhang, S. S.; Han, D. L. et al. Systemic delivery of microRNA for treatment of brain ischemia. Nano Res. 2021, 14, 3319-3328.

[13] Zhao, S. L.; Zhang, D. T.; Jiang, S.; Cui, Y.; Li, H. J.; Dong, J. C.; Xie, Z. R.; Wang, D.-W.; Amal, R.; Xia, Z. H. et al. Carbon-supported layered double hydroxide nanodots for efficient oxygen evolution: Active site identification and activity enhancement. Nano Res. 2021, 14, 3329-3336. 